Revue d'histoire de l'Amérique française

REVUE D.HISTOIRE DE L'AMÉRIQUE FRANÇAISE

\title{
La croissance de Montréal au XVIIIe siècle
}

\section{Louise Dechêne}

Volume 27, numéro 2, septembre 1973

URI : https://id.erudit.org/iderudit/303263ar

DOI : https://doi.org/10.7202/303263ar

Aller au sommaire du numéro

Éditeur(s)

Institut d'histoire de l'Amérique française

ISSN

0035-2357 (imprimé)

1492-1383 (numérique)

Découvrir la revue

Citer cet article

Dechêne, L. (1973). La croissance de Montréal au XVIIIe siècle. Revue d'histoire de l'Amérique française, 27(2), 163-179. https://doi.org/10.7202/303263ar d'utilisation que vous pouvez consulter en ligne.

https://apropos.erudit.org/fr/usagers/politique-dutilisation/ 


\title{
LA CROISSANCE DE MONTREAL AU XVIIIe SIËCLE *
}

\author{
LOUISE DeChÊNE \\ Département d'histoire \\ Université McGill
}

$\mathrm{Au}$ milieu du XVIII ${ }^{e}$ siècle, le Canada comptait environ $20 \%$ de citadins. La proportion, très élevée pour l'époque, semble indiquer que les villes de Québec et de Montréal exerçaient une attraction sur les campagnes et se peuplaient relativement plus vite que celles-ci. Quelques témoignages contemporains contribuent à renforcer cette impression ${ }^{1}$. Mais dans le contexte colonial, le rapport entre populations urbaine et rurale est un indice trompeur. Comme le commerce précède l'agriculture, ce rapport décroît avec le peuplement et tend à se stabiliser autour de $10 \%$, un point d'équilibre normal dans une économie préindustrielle. En raison de la faiblesse de l'immigration, la chute est beaucoup plus lente en Nouvelle-France qu'ailleurs et le nombre relatif de citadins n'est pas significatif.

Il faut examiner de plus près l'évolution démographique de ces villes, en particulier celle de Québec, la plus importante, qui présente peut-être quelques signes de dynamisme urbain, absents à Montréal. Nos recherches sur cette dernière région nous ont permis, malgré la carence des sources, de mesurer la progression de la population dans ce qui n'est encore qu'un poste complémentaire de l'intérieur et, même si nos conclusions ne peuvent être généralisées, Montréal offre un bon exemple de l'inertie qui guette une économie basée sur un "staple", que rien ne vient relayer. Le tableau 1 regroupe une série de chiffres approximatifs, car parmi les vingt-trois recensements de la population canadienne au XVIII ${ }^{\mathrm{e}}$ siècle, deux seulement, en 1707 et en 1754, isolent la population de Montréal de celle de la banlieue

* Une première version de ce texte a été présentée au colloque Historical Urbanization in North America, tenu à l'Université York du 24 au 26 janvier 1973.

1 C'est celle qu'ont retenue, entre autres, Guy Frégault, La civilisation de la Nouvelle-France 171s-1744 (Montréal, 1969), 168 et "La NouvelleFrance, territoire et population", dans Le XVIIIe siècle canadien. Etudes (Montréal, 1968), 48-49; Louis Trottier, "La genèse du réseau urbain du Québec", dans L'urbanisation de la société canadienne-française (Québec, 1968), 23.

RHAF, vol. 27, no 2 (septembre 1973) 


\section{TABLEAU 1}

Estimation de la population de Montréal et croissance comparée

MONTRÉAL:

VILLE ET FAUBOURGS
CANADA (BAS-CANADA)

$\begin{array}{ccc}\text { Taux, } & \begin{array}{c}\text { Taux, } \\ \text { accrois- }\end{array} \\ \text { sement } & \begin{array}{c}\text { Nombre } \\ \text { d'habi- }\end{array} & \begin{array}{c}\text { accois- } \\ \text { sement }\end{array}\end{array}$
annuel tants annuel

\begin{tabular}{|c|c|c|c|c|c|c|}
\hline Années & Maisons & Ménages & $\begin{array}{l}\text { d'habi- } \\
\text { tants }\end{array}$ & $\begin{array}{l}\text { sement } \\
\text { annuel }\end{array}$ & $\begin{array}{l}\text { d'habi- } \\
\text { tants }\end{array}$ & $\begin{array}{l}\text { sement } \\
\text { annuel }\end{array}$ \\
\hline 1697 & 152 & - & $(1150)^{\mathrm{a}}$ & - & $(13,200)^{b}$ & - \\
\hline 1707 & - & 204 & 1327 & $1.4 \%$ & 17,615 & $2.9 \%$ \\
\hline 1731 & 400 & - & $(2980)^{c}$ & $3.4 \%$ & $(34,850)^{c}$ & $2.9 \%$ \\
\hline 1739 & - & - & $(3450)^{d}$ & $1.7 \%$ & 43,264 & $2.7 \%$ \\
\hline 1741 & 457 & 591 & $(3575)$ & - & - & - \\
\hline 1754 & - & - & 4000 & $0.8 \%$ & 55,000 & $1.6 \%$ \\
\hline 1781 & 659 & - & $(5300)$ & - & - & - \\
\hline 1784 & - & - & $(5500)^{e}$ & $1.0 \%$ & 113,012 & $2.5 \%$ \\
\hline 1831 & 3774 & - & 27,297 & $3.5 \%$ & 553,134 & $3.4 \%$ \\
\hline
\end{tabular}

Sources: Livre des tenanciers de Montréal, 1697, Arch. St-Sulpice; recensements du Canada, AC, G1, 460-1; Recensement du Canada, 1871, IV; dénombrement seigneurial de 1731, RAPQ (1941-42); perquisition de 1741, MSRC, III, XV (1921); C. Perrault, Montréal en 1781; recensement de 1784, W. Kingsford, The history of Canada, 7:204; recensement de 1831, $J A L B C, 41$, app. 0 o.

Notes du tableau:

a A raison de sept personnes par maison, rapport obtenu en 1731. Nous additionnons ensuite le personnel des communautés religieuses.

b Moyenne, d'après les recensements de 1695 et 1698.

c Moyenne entre les recensements de 1730 et 1732 .

d Population recensée dans la paroisse Notre-Dame, moins 900 habitants, estimation minima pour la banlieue.

e La population globale de la paroisse est de 6479 habitants. D'après le dénombrement seigneurial de 1781 , il y a encore 120 feux ruraux. Nos estimations de la population urbaine en 1781 et 1784 sont certainement des maximums, car elles correspondent à un rapport de huit personnes par maison, une densité déjà un peu trop forte pour cette période. John Hare a obtenu un rapport de 7.2 personnes dans la ville de Québec en 1795 et à la fin du XIX ${ }^{\mathrm{e}}$ siècle il n'y a que 8 personnes par maison dans un quartier ouvrier de Montréal. Voir "La ville de Québec, 1792-1805: étude socio-professionnelle", communication présentée au GRISCAF en novembre 1971; H. B. Ames, The City below the hill (Toronto, 1972). 
essentiellement agricole comprise dans la même paroisse. En utilisant quatre dénombrements des domiciles dressés en 1697, 1731,1741 et 1781 , il est possible d'évaluer le nombre de ces ruraux - un groupe assez stable qui oscille un peu en deçà de mille habitants - et de le défalquer des données globales, pour ne retenir que la population de la ville et des faubourgs. La compilation des recensements publiée en 1871, qui a servi de base à la monographie sur la composition urbaine et rurale de la population canadienne, publiée en 1931, et à d'autres travaux postérieurs, avance quelques chiffres hautement fantaisistes, notamment 18,000 habitants à Montréal en $1790 .^{2}$ L'utilisation de toutes les données quantitatives, jointe à de multiples recoupements qu'il serait fastidieux de retracer ici, nous autorisent à croire que nos estimations sont beaucoup plus proches de la réalité. Tout au long du XVIII siècle, après comme avant la Conquête, Montréal se développe à un rythme très lent. Ce sont les aspects et les implications de cette croissance que nous analysons brièvement. ${ }^{3}$

\section{L'occupation de l'espace urbain}

Ceux qui fondèrent et habitèrent Montréal ne désignèrent jamais autrement que par le mot "ville" l'espace de 110 arpents réservé à cette fin vers 1648 et assez tôt délimité par une palissade. Même si la réalité d'alors, quelques champs et une poignée d'habitants autour d'un magasin, ne correspond guère à nos définitions, nous retenons le mot. Ces colons ne parlèrent jamais de bourg ou de village. Ils avaient l'impression de créer une ville.

Jusque vers 1665-1670, Montréal est un comptoir, ou centre d'échange entre deux peuples de civilisation différente, au milieu d'une zone agricole en expansion. La traite des fourrures à l'intérieur du comptoir implique une participation, inégale sans doute, mais participation quand même, de toute la population,

${ }^{2}$ Recensement de 1871, IV; Census of 1981, Monograph 6, "Rural and Urban Composition of the Canadian Population" par S. A. Cudmore et H. G. Caldwell.

${ }^{3}$ Nous laissons de côté d'autres aspects de l'histoire de Montréal, institutions, organisation politique et sociale, etc., qui ont été abordés dans les ouvrages suivants: W. H. Atherton, Montreal 1535-1914 (Montréal, 1914) ; C. Bertrand, Histoire de Montréal (Montréal, 1935-42) ; J. I. Cooper, Montreal. A brief History (Montréal, 1969); R. Rumilly, Histoire de Montréal (Montréal, 1970) I-II; E. R. Adair, "The Evolution of Montreal under the French Regime", CHAR (1942) et autres, parmi lesquels il faudrait citer tous les travaux de O. Maurault et de E.-Z. Massicotte. 
soit environ 200 "habitants" ${ }^{4}$ à la fin de cette période. Le lieu désigné pour la ville est celui de la première agriculture, pratiquée sur des parcelles allant de quelques centaines de pieds à deux et trois arpents, après quoi les champs commencent à déborder dans les côtes environnantes. Les activités agricoles et commerciales sont d'abord fortement imbriquées. Les colons qui reçoivent des terres dans la périphérie s'attardent dans l'espace occupé, où ils trouvent à la fois une protection et des profits faciles, qu'ils appliquent avec plus ou moins de zèle au défrichement. De leur côté, presque tous les traitants et les artisans, excités par les hauts prix des produits agricoles, engagent des serviteurs pour mettre leur terre en valeur. La première agglomération répond avant tout à des impératifs défensifs, car la nature des échanges n'exigeait rien de plus qu'un entrepôt et une petite base agricole. Les traitants avaient même avantage à se disperser et, sans la menace qui pesait sur la colonie, un réseau de comptoirs aurait fort bien pu s'organiser. Déjà, en amont des rapides, à Lachine, Châteauguay, sur le lac desDeux-Montagnes, des postes sont créés dès que le danger des attaques iroquoises s'estompe.

Le bouleversement de cette structure commerciale initiale favorise le développement de Montréal. La récession dans le marché européen des fourrures, qui réduit les marges de profit, liée aux manœuvres des marchands de Québec pour combiner le commerce d'importation avec l'échange direct qui leur échappe, engagent les Canadiens dans une course séculaire vers la source des fourrures ${ }^{5}$. La tendance à la dispersion du commerce à l'intérieur de la colonie, qui commençait à s'esquisser, est bloquée dès que les Indiens cessent d'y descendre leurs fourrures. C'est à ce moment que les remarquables avantages de la situation de Montréal commencent à jouer pleinement ${ }^{\circ}$. La ville est pour ainsi dire recréée autour d'une nouvelle fonction dominante: la circulation des fourrures et des marchandises de traite entre Québec et l'intérieur du continent. Elle est non seulement le point de transbordement, mais l'endroit où s'opère le changement de propriété. La nouvelle organisation commerciale entraîne un réaménagement progressif de l'espace. Les champs sont lotis

4 On appelle habitants à cette époque les hommes libres propriétaires d'un bien-fonds dans la colonie, ce qui ne comprend donc pas les engagés et les soldats. Le recensement de 1666 énumère 659 personnes dans l'île de Montréal, dont 141 habitants, chefs de ménage.

5 H. A. Innis, The Fur Trade in Canada: An Introduction to Canadian Economic History (Toronto, 1956).

6 Raoul Blanchard, L'Ouest du Canada français. Montréal et sa région (Montréal, 1953). 
pour accommoder les professionnels de la traite et l'armature artisanale complémentaire. La hausse de la valeur foncière incite des habitants à vendre leurs emplacements et chassent ceux qui n'étaient que locataires ou squatters. Désormais tout à fait exclus du commerce, les colons se dispersent dans les côtes.

Mais la concentration urbaine qui résulte de cette redéfinition est limitée et il faut d'autres fonctions, religieuse, militaire, administrative, services à la communauté rurale, pour soutenir la croissance qui demeure néanmoins inférieure à celle de l'ensemble de la colonie jusqu'à la fin du siècle. Si Montréal se gonfle subitement dans la dernière décennie, c'est qu'elle devient momentanément la ville refuge. Des campagnes environnantes, des seigneuries de la rive sud, les habitants ruinés ou simplement apeurés par les raids anglo-iroquois affluent dans l'enclos urbain, qui offre encore de vastes terrains déserts où chacun peut se construire un abri temporaire. Une liste des propriétés dressée à la fin de cette période "d'encabanement" montre que, malgré ses 152 maisons, Montréal est encore un établissement très primitif.

C'est dans le premier quart du XVIII ${ }^{e}$ siècle que le développement urbain s'affirme avec le plus de vigueur. La ville retient un fort pourcentage de soldats démobilisés ${ }^{7}$ et attire des Canadiens. Ceux-ci ne sont pas des ruraux cependant, mais principalement des traitants, artisans et manœuvres de Québec et de Trois-Rivières ${ }^{8}$. Ces arrivages ne correspondent pas à une expansion du commerce. Bien au contraire, ils s'inscrivent dans le cœur d'une crise de surproduction et d'avilissement des prix du castor et dans la très lente remontée qui suit. C'est un mouvement de remplissage qui correspond à la mise en chantier de travaux publics, soit la construction d'une muraille autour de la ville. L'agiotage, qui accompagne la crise monétaire et suscite vers 1710-1720 une vague sans précédent d'investissements immobiliers, n'est pas non plus étranger à cet essor ${ }^{9}$. Le secteur du bâtiment bénéficie surtout de ce sang nouveau et nous voyons aussi apparaître quelques métiers proprement urbains, tels que perruquiers, orfèvres, jardiniers, etc.

7 L'île de Montréal accueille plus de quatre cents soldats démobilisés entre 1697 et 1715 , la plus forte vague d'immigration depuis la fondation, dont la ville bénéficie en partie. Dame.

8 D'après le lieu d'origine des époux: registres de la paroisse Notre-

${ }^{\circ}$ La crise est catastrophique pour l'Etat, mais non pas pour les marchands et la colonie en général. Jean Hamelin en a fait un portrait très sombre dans Economie et Société en Nouvelle-France (Québec, 1960), 37-46. 
La première image de Montréal date de 1731: 379 maisons dans l'enceinte de la ville, la majorité en bois, très basses, semblables aux demeures rurales; $44 \%$ sont construites en pierres et le quart, à deux étages. Les familles à revenus modestes ne disposent que d'une pièce ou deux sous le grenier, la boutique de l'artisan comprise, si elle n'est pas installée dans un appentis; une seule cheminée, soit une unique "chambre à feu". A un niveau d'aisance supérieur, la maison de maçonnerie est plus spacieuse, environ cinquante par trente pieds, et beaucoup plus confortable: deux cheminées, deux à trois pièces par étage et des cabinets attenants aux chambres communes. Chez les marchands, le magasin est à l'étage et la boutique au rez-de-chaussée, plus rarement dans un autre corps de logis. Grains et fourrures sont gardés au grenier. Il reste quatre ou cinq pièces à l'usage de la famille, ce qui fait, à raison de 5.5 personnes par famille plus deux domestiques, une moyenne de 11/2 personne par chambre, soit deux fois plus d'espace que chez les gens du commun ${ }^{10}$.

Il y a cinq couvents et cinq églises et chapelles. Les édifices publics et les bâtiments privés à fins commerciales et industrielles se résument à deux écoles, un tribunal, une prison, un corps de caserne, une canoterie, une brasserie et sept entrepôts. Les remparts non encore achevés comptent plusieurs portes et bastions. Le plan urbain est régulier et aéré. Deux rues principales de vingt-quatre pieds de large s'allongent parallèlement au fleuve et sont coupées à angle droit par une douzaine de rues plus étroites. Des tronçons d'artères au bord de l'eau et en haut de la butte brisent un peu l'ordonnance en damier. La place du marché est bordée par les résidences des principaux habitants. Les propriétés conventuelles et autres réserves appartenant à la paroisse, au gouverneur, créent çà et là de larges espaces vides, mais leur présence n'étrangle pas la ville, à preuve ses cent soixante-six jardins. Les rues non pavées sont sales et boueuses et, comme la ville est petite et les chemins qui l'entourent rarement praticables, les bourgeois n'ont pas de voitures de promenade. Les écuries sont exceptionnelles, mais derrière les maisons nous trouvons généralement un hangar et une étable qui abrite quelques animaux, des porcs surtout ${ }^{11}$. A l'ouest de la porte des Récollets, le faubourg Saint-Joseph ne

10 Dans les villes françaises de cette époque, la moyenne serait d'une pièce par famille de 4.5 personnes: $\mathrm{F}$. Braudel et $\mathrm{E}$. Labrousse, Histoire économique et sociale de la France, 1660-1789 (Paris, 1970), 49.

11 Voir toutes les ordonnances de police touchant la malpropreté, les porcs en liberté, les dangers d'incendie, etc.; Arch. judiciaires de Montréal. Ce sont des traits communs à toutes les villes, petites et moyennes, du XVIIIe siècle. 
compte encore que vingt-et-une maisons, deux entrepôts et les bâtiments de l'Hôpital Général.

Un autre dénombrement effectué dix ans plus tard permet de saisir le rythme de l'évolution. Même en tenant compte d'un incendie, qui dans l'intervalle a rasé quarante-six immeubles ${ }^{\mathbf{1 2}}$, il est étonnant de ne trouver que 387 maisons dans l'enceinte urbaine, ce qui équivaut à 5.4 constructions par année seulement. $\mathrm{La}$ ville semble figée. Trois faubourgs, à l'ouest, au nord et à l'est, totalisent soixante-dix maisons. $70 \%$ des 591 ménages compris dans l'ensemble de l'agglomération sont propriétaires. Les nombreux baux de location contenus dans les minutes notariales et la présence de ces 176 locataires nous ont fait croire un instant à une certaine concentration de la propriété, l'indice d'une conjoncture urbaine favorable. Il n'en est rien. Seulement vingt-huit individus ou communautés sont propriétaires de deux maisons ${ }^{13}$; trente-trois propriétaires partagent leur demeure avec les locataires; en tout soixante-six maisons logent deux familles et plus. La densité à l'intérieur des murs, soit 32.5 personnes à l'acre, reste très faible. Bien que fort incomplète, la nomenclature des métiers montre que les marchands et autres notables forment un îlot au cœur de la ville, mais dès qu'on s'en éloigne, d'autres marchands et des officiers voisinent avec les artisans et des individus non identifiés, vraisemblablement de condition modeste. Seule la rue Saint-Jacques a un caractère artisanal prononcé ${ }^{14}$. Bref, c'est une impression de médiocrité assez uniforme qui se dégage de ce recensement.

TABLEAU 2

Répartition des maisons dans la ville et les faubourgs

\begin{tabular}{cccc} 
& \multicolumn{2}{c}{ NoMBRE DE MAISONS } & \\
Années & Ville & Faubourgs & Total \\
1731 & 379 & 21 & 400 \\
1741 & 387 & 70 & 457 \\
1781 & 307 & 352 & 659
\end{tabular}

12 Soit l'incendie de 1734 , la seconde conflagration à Montréal. Celle de 1721 aurait détruit cent cinquante maisons, qui furent vite rebâties: M. Mondoux, L'Hôtel-Dieu premier hôpital de Montréal... (Montréal, 1942), 271-2 et 289.

13 La concentration foncière est peut-être légèrement supérieure à ce qu'il paraît, car les gens bâtissent parfois sur des terrains loués, les petites maisons de bois étant facilement démontables.

14 Dans toutes nos listes, les habitants sont très mal identifiés, parfois seulement par leur surnom. Le travail de E.-Z. Massicotte sur le dénombrement de 1741 nous a beaucoup facilité la tâche: $M S R C$, III, XV (1921): $1-61$. 
Le dénombrement seigneurial de 1781 présente un cas intéressant de réorganisation spatiale. En 1765 , la ville a été à nouveau partiellement détruite par un incendie et, à la faveur de la reconstruction, elle s'est transformée. A l'intérieur des remparts, il n'y a plus que 307 maisons, soit $20 \%$ de moins qu'en 1741. Ce sont de grandes demeures de deux et trois étages, presque toutes en pierres. Sur la majorité des terrains, s'élèvent des dépendances en maçonnerie, dont plusieurs écuries. Notons également ces structures que le recenseur appelle "voûtes en plafonds", sortes d'entrepôts surélevés au-dessus de la cour. Les terrains, plus vastes qu'antérieurement, abritent encore des jardins et sont généralement clos par une muraille. Montréal a perdu son allure de grand village dans lequel pauvres et riches vivaient pêle-mêle. Elle a été réaménagée pour servir essentiellement de centre résidentiel et commercial à la classe bourgeoise ${ }^{15}$. Si l'incendie a facilité cette évolution, d'autres facteurs ont joué pour pousser les petits artisans et les manœuvres, sinistrés et non sinistrés, hors des murs. Prospérité nouvelle, enchérissement du terrain, augmentation de la valeur locative, distribution plus inégale des revenus, etc. Les ouvrages qui couvrent cette période ne nous permettent pas de démonter le mécanisme de cette ségrégation prématurée ${ }^{16}$. Nous observons seulement que la déconcentration, phénomène normal dans le processus d'urbanisation, s'est produite ici en l'absence de toute pression démographique, comme en témoigne la croissance médiocre des faubourgs.

Pour mieux les encadrer, nous avons rattaché nos estimations au recensement de 1831, mais nous n'entendons pas aborder ici les transformations majeures qui marquent le premier quart du XIX ${ }^{\mathrm{e}}$ siècle dans le Bas-Canada ${ }^{17}$. Il faudrait chercher quelle est l'influence respective du progrès économique enregistré au

15 Il n'y a que 45 propriétaires d'origine britannique ou américaine. Le reste, soit $82 \%$, sont Canadiens, la plupart citadins depuis plusieurs générations.

16 A Boston, une ville qui se développe rapidement, déjà en 1774 les pauvres ont été repoussés vers la périphérie: S. B. Warner, "A Scaffolding for Urban History", dans A. M. Wakstein, ed., The Urbanization of America. An Historical Anthology (Boston, 1970), 60; sur ces mêmes problèmes, à un stade plus avancé de croissance, voir: L. F. Schnore et P. R. Knights, "Residence and Social Structure. Boston in the Ante Bellum Period", dans S. Therstrom et R. Sennett, eds., Nineteenth Century Cities. Essays in the New Urban History (Yale U.P., 1969), 247-256.

17 Voir Fernand Ouellet, Histoire économique et sociale du Québec, 1760-1850 (Montréal, 1966) et les nombreux articles de Jean-Pierre Wallot et Gilles Paquet sur la période 1790-1815. 
début du siècle et de l'immigration massive qui commence après 1815 dans cette accélération de la croissance urbaine.

\section{Le bilan}

Trop d'éléments manquent encore pour unir dans une même équation la croissance réelle de Montréal, le mouvement naturel de sa population et l'évolution démographique dans l'ensemble de la colonie. Nous nous bornons à souligner les problèmes et les obstacles. En 1731, la paroisse Notre-Dame compte presque un quart de ruraux, ce qui empêche de bien dégager les comportements démographiques urbains. Mais même en faisant abstraction de cette difficulté, il serait futile de calculer des taux de natalité et de mortalité à partir d'effectifs aussi faibles, constamment secoués par les phénomènes migratoires qui font varier à court terme la structure d'âges. Seule la reconstitution des familles sur une longue période donnerait de bons résultats, mais en attendant, de simples comptages dans les registres paroissiaux peuvent suggérer les tendances. Or ceux que nous avons faits dans les cinq paroisses de l'île de Montréal avant 1715 montrent qu'il n'y a pas, à première vue, de régime démographique différentiel entre ville et campagnes. Par exemple, le mouvement saisonnier des mariages et des conceptions est le même, tout comme les rapports grossiers établis entre les mariages et les naissances ${ }^{18}$. Les épidémies ne semblent pas être plus meurtrières dans la paroisse urbaine, si l'on excepte les soldats, qui de toute manière ne sont jamais recensés avec la population civile. Ces constatations sont peut-être encore valables au XVIII ${ }^{\mathrm{e}}$ siècle, car les conditions gênérales, densité et niveau de vie, ne changent guère.

Il est donc possible d'utiliser les données démographiques générales de la colonie, mais elles sont incomplètes. Il n'y a pas de statistiques de l'immigration en Nouvelle-France. Le bilan migratoire qui a été retenu, environ 10,000 personnes réparties inégalement entre 1608 et 1760 , est simplement le solde entre l'accroissement réel et le mouvement naturel connu d'après les registres paroissiaux. Tout le problème de l'émigration reste dans l'ombre: celle des Canadiens vers l'Ouest, la Louisiane et la France, celle des nouveaux venus qui retournent dans leur pays après un court séjour. Ces départs sont assez nombreux

${ }^{18}$ Le rythme de la vie est fortement conditionné par la nature même dans la ville et notons d'ailleurs qu'il y a coïncidence entre les travaux des champs et les voyages de traite. Quant au rapport entre naissances et mariages, il est de l'ordre de 5 dans toutes les paroisses. 
dans la couche supérieure de la société, marchands et officiers, et peut-être davantage chez les petites gens. Par exemple, selon les périodes et le mode de recrutement, entre 25 et $50 \%$ des engagés qui passent à Montréal au XVII ${ }^{\mathrm{e}}$ siècle retournent en France ${ }^{19}$. Or ce va-et-vient est certainement plus sensible dans les villes.

La qualité des statistiques de l'état civil pose un autre problème. Nous connaissons, grâce aux travaux de Jacques Henripin, la nuptialité et la fécondité de cette population ${ }^{20}$, mais la mortalité générale n'a pas encore fait l'objet d'une étude attentive. Le sous-enregistrement des décès a déjà été bien démontré par Hubert Charbonneau et ses collègues ${ }^{21}$ et nos comptages révèlent les mêmes lacunes. Il suffit de comparer les taux de mortalité infantile obtenus d'après les décès enregistrés, variant entre 10 et $18 \%$ à la fin du XVII ${ }^{\mathrm{e}}$ siècle, avec le taux de $24.6 \%$ établi solidement par Henripin ${ }^{22}$, pour douter de ces taux de mortalité générale qui seraient inférieurs à trente pour mille avant 1740 . Par suite, le taux de croissance naturelle, qui se maintiendrait autour de $3 \%$ pendant tout le $\mathrm{XVIII}^{\circ}$ siècle, devient très fragile ${ }^{23}$.

Bref nous devons nous contenter d'observer que, sauf pour une courte période, la croissance de Montréal est beaucoup plus lente que celle de l'ensemble de la colonie. Il est certain que des taux d'accroissement réel de 0.8 et $1 \%$ resteraient toujours en deçà de la croissance naturelle, même si celle-ci était rajustée en fonction d'une mortalité plus forte, voire même d'une natalité urbaine légèrement inférieure. En supposant nuls les arrivages

$19 \mathrm{Ce}$ qui est encore un bilan très positif si nous comparons par exemple avec le Maryland où seulement $7 \%$ des serviteurs importés entre 1670 et 1680 peuvent être retracés par la suite dans la colonie: A. E. Smith, Colonists in Bondage, 298-9.

${ }^{20}$ La population canadienne au début du XVIIIe siècle. Nuptialité, fécondité, mortalité infantile (Paris, 1954).

${ }^{21} \mathrm{H}$. Charbonneau, J. Légaré et Y. Lavoie, "Recensements et registres paroissiaux du Canada durant la période 1665-1668: étude critique", Population, XXV, 1 (janv.-fév. 1970) : 97-124.

22 Op. cit.: 103-107.

$23 \mathrm{~J}$. Henripin, Tendances et facteurs de la fécondité au Canada (Ottawa, 1968), 5-8. Les taux de mortalité générale par décennie seraient de 24 et 25 pour mille entre 1700 et 1740 , de 32 à 34 pour mille entre 1740 et 1790 et ne descendent en-dessous de 27 pour mille qu'après 1840 . La structure par âges, une pyramide très large à la base et vite étriquée, ne change guère tout au long du XVIII e siècle et il n'y a pas lieu de croire que la mortalité en 1700 était inférieure à celle enregistrée en 1800 . C'est la tenue des registres paroissiaux qui s'améliore. 
d'immigrants dans la ville après 1731 , ce qui n'est pas le cas ${ }^{24}$, néanmoins l'image d'une déperdition constante s'impose. Bien que les travaux sur la démographie urbaine de la France de l'ancien régime soient encore peu nombreux, il semble acquis que la croissance des villes est généralement plus faible que celles des campagnes prospères ${ }^{25}$. Le fléchissement de la natalité, la mortalité accrue y engendrent un déficit endémique qui n'est pas toujours entièrement comblé par l'apport rural. Le retard de Montréal sur les campagnes résulte d'un processus différent: ici la ville enregistre un excédent naturel, qu'elle rejette en partie, et il semble exclu qu'elle ait pu exercer la moindre attraction sur les ruraux.

\section{Aspects de l'économie urbaine}

La faiblesse du développement urbain est liée à l'économie générale du pays et surtout au rôle de la ville dans cette économie. Montréal cumule plusieurs fonctions et, pour mesurer l'importance de chaque secteur, il faudrait commencer par dresser un tableau des occupations et de leurs effectifs respectifs. Les sources ne permettent pas d'être aussi rigoureux, mais nous avons suffisamment fréquenté cette société et dépouillé tous les documents disparates qui la concernent - minutes notariales et judiciaires, rôles d'impositions, etc. - pour présenter une sorte d'inventaire des activités urbaines vers 1731 .

Le commerce des fourrures a été le principal facteur de création de Montréal, mais, une fois la charpente élémentaire mise en place, il n'est pas un facteur de développement. Après 1731 , la croissance de la ville ralentit, alors que le volume des exportations ne cesse d'augmenter. L'entrepôt et l'expédition exigent peu de main-d'œuvre. Ce commerce soutient environ trente marchands et une vingtaine d'autres vivent précairement du marché local et d'équipements de traite irréguliers. Les voyageurs sont les descendants des meilleurs coureurs de bois, naguère d'origine rurale, mais peu à peu ils s'associent plus étroitement aux marchands, acquièrent une place enviable dans la société, fondent des familles qu'ils installent dans la ville. Dans

${ }^{24} \mathrm{Il}$ y a une forte vague d'immigration dans la colonie entre 1740 et 1760: P. E. Renaud, Les origines économiques du Canada. L'ouvre de la France (Paris, 1928).

${ }^{25}$ Dans le cas des villes de l'intérieur, petites et moyennes, et non pas dans les grands ports comme Nantes, Bordeaux et Marseille: P. Goubert, "Révolution démographique au XVIII siècle", dans F. Braudel et E. Labrousse, op. cit., 72-74. 
ce groupe, la tendance est à la concentration; les effectifs n'augmentent pas, le recrutement se fait par l'intérieur et par le haut. Il y a peut-être soixante-dix ménages de voyageurs à Montréal en 1731 et plusieurs commencent à émigrer en permanence vers Détroit et Michilimakinac. Chaque année, les marchands et voyageurs embauchent entre 250 et 300 jeunes gens de la colonie pour le voyage saisonnier dans l'Ouest. Les citadins sont proportionnellement mieux représentés que les ruraux dans ces contingents d'engagés, mais l'offre de main-d'œuvre est bien supérieure à la demande et tous les garçons n'ont pas la vigueur nécessaire pour avironner jusqu'au lac Supérieur et portager des ballots de cent livres. Ce n'est sans doute pas plus du tiers de la jeunesse de la ville qui réussit à se faire embaucher. C'est dans ce groupe que s'exerce la plus forte ponction migratoire. Une cinquantaine d'individus, aubergistes, cabaretiers, charretiers, boulangers et bouchers, doivent une partie de leur clientèle au commerce des fourrures, lequel entretient aussi, bien maigrement, un grand nombre de couturières à domicile. Mais là encore, les commandes sont insuffisantes par rapport au travail disponible et aux besoins. Au total c'est environ le tiers de la population active qui subsiste inégalement grâce au grand commerce.

Il n'y a pas d'industries à Montréal. Peu d'ateliers emploient plus de deux ou trois apprentis, même en comptant les scieries et tanneries qui opèrent dans la banlieue. Une cinquantaine d'artisans travaillent pour le marché local, urbain et rural. Les armuriers, forgerons, tonneliers et menuisiers exécutent bien quelques commandes pour les traitants, mais pour la majorité, c'est une source de revenus marginale. Le divorce entre le capital marchand et la production artisanale est la règle. L'ouvrier est propriétaire de ses outils et de la matière première et vend directement aux consommateurs. Le marchand n'essaie pas de resserrer les liens de dépendance, qui naissent occasionnellement de l'endettement, pour pousser la production et amorcer des rapports capital-travail ${ }^{26}$. Le marché urbain croît à peine et le marché rural desservi par les artisans de la ville est limité par les centres élémentaires qui sont créés çà et là dans l'arrièrepays, en réponse à l'expansion de la population rurale. Une fois assuré le strict remplacement des gens de métier, leurs fils ne

${ }^{26}$ Ces observations reposent sur l'analyse des comptes des marchands de Montréal. Sur ces questions, voir Maurice Dobb, Etudes sur le développement du capitalisme (Paris, 1969). 
peuvent qu'augmenter la masse des journaliers ${ }^{27}$. Les métiers du bâtiment rassemblent environ la moitié de la main-d'œuvre spécialisée et occupent les manœuvres. Mais, encore en 1731, presque la moitié des maisons ne sont que de simples assemblages de pièces de bois, que les propriétaires construisent euxmêmes. La construction domiciliaire plus élaborée est lente. Celle des églises et des couvents, avec les travaux publics ${ }^{28}$, créent quelques chantiers plus importants au cours du siècle, mais tous ces travaux sont interrompus au moins cinq mois par année. Il y a donc une situation de chômage chronique, à laquelle les nouvelles générations de citadins et les immigrants, un instant attirés par la ville, répondent en quittant le pays ou en s'installant sur une terre.

Ensemble, les services administratifs, religieux, militaires et domestiques peuvent occuper les deux-cinquièmes de la population active. Montréal compte relativement peu de fonctionnaires, mais, comme dans toutes les villes françaises de l'ancien régime, il y a inflation du personnel religieux ${ }^{29}$. Ici, c'est surtout un phénomène féminin qui touche les classes aisées, la conséquence évidente de l'émigration masculine. Au XVII ${ }^{\circ}$ siècle, l'évangélisation a aussi été un facteur de création urbaine. Elle apporta des capitaux importants ${ }^{30}$ et, avec toute la détermination et la persévérance qu'elle suppose, constitua un pôle de stabilité à une époque difficile. Mais, avec le temps, les effectifs conventuels tendent à augmenter plus rapidement que les services qu'ils dispensent ${ }^{31}$.

Montréal est une ville de garnison. La population militaire cantonnée à l'intérieur des murs et aux alentours varie selon les

27 La pénurie de gens de métier au Canada est une conséquence et non pas une cause du marasme économique. Ainsi Montréal doit aller chercher à Québec des ouvriers spécialisés pour ses gros chantiers, précisément parce que la demande est trop irrégulière pour les entretenir sur place. Voir Pierre Harvey, "Stagnation économique en Nouvelle-France", L'Actualité économique, 37: 537-548; aussi, J. Mathieu, La construction navale royale à Québec, 1739-1759 (Québec, 1971).

28 Il faut des hommes de métier pour diriger les chantiers, mais, dans le cas de l'église et des remparts, une partie de la main-d'œuvre est fournie par les contribuables qui versent leur quote-part en journées d'ouvrage.

29 Soit une centaine de religieuses et trente à quarante ecclésiastiques.

30 Capitaux français et une partie du budget de la colonie que ces œuvres réussissent à drainer vers Montréal sous forme de subventions régulières: L. Gérin, Aux sources de notre histoire: les conditions économiques et sociales de la colonisation en Nouvelle-France (Montréal, 1946), 162-191.

31 Notons le contraste entre cette pléthore de religieuses et de réguliers et la pénurie de prêtres pour desservir les paroisses rurales. 
périodes. Vers 1731, il peut y avoir quelque deux cents soldats logés chez l'habitant et dans la caserne ${ }^{32}$. En termes de consommation, cette présence est sans doute favorable à la ville, mais, depuis la fin du $\mathrm{XVII}^{\mathrm{e}}$ siècle, elle joue un rôle néfaste dans la distribution de l'emploi. L'administration tolère que les soldats travaillent à la journée à des gages inférieurs à ceux des civils, concurrence malencontreuse pour ces derniers ${ }^{33}$. Si la troupe est d'origine française, ses officiers sont en majorité des Canadiens à cette époque. Près d'une quarantaine sont domiciliés à Montréal, soit l'état-major du gouvernement et ceux qui y laissent leur famille lorsqu'ils servent dans les postes éloignés. La plupart sont étroitement associés au commerce des fourrures et nous pourrions fort bien les inclure dans le personnel de la traite.

Le service domestique est un débouché important pour la main-d'œuvre urbaine. Les serviteurs étaient relativement moins nombreux en 1731 qu'à la fin du siècle, mais les données précises du recensement de 1784 illustrent une situation déjà ancienne: le sous-emploi. A cette dernière date, les domestiques (apprentis compris, semble-t-il) forment presque le cinquième de la population urbaine globale. Le groupe comprend 142 esclaves, 129 enfants de moins de quinze ans et 913 adultes, hommes et femmes à peu près également représentés ${ }^{34}$. A une époque où la mise en service des enfants était partout coutumière, rares sont ceux de Montréal qui quittent leur famille ${ }^{35}$ et, si les bourgeois emploient surtout des adultes, c'est que ceux-ci acceptent des gages modestes. Les esclaves indiens qui apparaissent au début du XVIII

32 Les soldats, célibataires recrutés en France, ne sont pas recensés, donc non compris dans nos estimations. En temps de paix, si leur capitaine y consent, ils peuvent se faire licencier à la condition de devenir colons. Plusieurs préfèrent terminer leur service et rentrer dans leur pays : Mémoire de R. d'Auteuil, Arch. des Colonies, C11A:34; E. Salone, La colonisation de la Nouvelle-France. Etude sur les origines de la nation canadiennefrançaise (rééd. 1969), 339-342.

33 Ce faisant, le soldat renonce à sa solde mais y gagne; son capitaine, qui garde la solde, fait une affaire. W. J. Eccles écrit: "The men provided a sorely needed pool of labour": "The Social, Economic and Political Significance of the Military Establishment in New France", CHR (mars 1971): 5-6. Ce qui était vrai au tout début de la colonie, quand les colons n'avaient pas encore de grands enfants pour les aider aux champs, ne vaut plus par la suite. Ce système de prévarication a surtout des effets nuisibles. $34 \mathrm{~W}$. Kingsford, op. cit., 7: 204. Ces chiffres doivent être rapportés à la population totale de la paroisse, soit 6479 habitants.

35 En 1689, dans la petite ville de Bristol en Nouvelle-Angleterre, un enfant sur cinq est en service. A Montréal en 1784, un sur quatorze seulement. John Demos, $A$ Little Commonwealth. Family Life in Plymouth Colony (New-York, 1970), 69-74. 
siècle ne coûtent pas cher, mais leur espérance de vie est faible ${ }^{36}$, de sorte qu'il n'y a pas avantage à les substituer à une maind'œuvre locale toujours abondante. Tel est donc le partage des occupations à Montréal pendant au moins un siècle. Le secteur productif figure en toute dernière place.

Les relations ville-campagnes sont complexes et nous ne soulignons ici que quelques aspects. Montréal n'est pas situé au centre d'une région agricole, mais sur la frange de l'espace seigneurial exploité, lequel demeure dans une large mesure l'hinterland de Québec. Les blés que le Canada commence à exporter au XVIII ${ }^{\mathrm{e}}$ siècle sont dirigés tout droit vers le port d'embarquement et cette timide intégration des campagnes au marché extérieur n'a aucune répercussion sur le développement de Montréal. Pour que la ville serve de relais au produit agricole, il faut ouvrir les terres en amont, ce qui deviendra le Haut-Canada. En attendant, Montréal entretient des relations assez ténues avec un arrière-pays étroit. Les campagnes des alentours nourrissent la ville et fournissent le biscuit pour l'Ouest. Parce que la demande extérieure a été longtemps inexistante et reste encore faible et capricieuse, les côtes tendent à se replier sur ellesmêmes et à échapper, autant que faire se peut, à des échanges denrées/marchandises dont les termes jouent contre elles ${ }^{37}$. Les paysans ne s'enrichissent pas, mais ils ne sont jamais vraiment affamés et la ville ne sert pas ici d'exutoire à la misère rurale. Il est inutile de spéculer sur la diversification économique qu'une politique moins généreuse envers les colons aurait rendue possible ${ }^{38}$. L'alourdissement des charges ne pouvait que rétrécir davantage le marché domestique, mais, les circonstances extérieures et les conditions démographiques demeurant les mêmes, nous ne voyons guère à quoi aurait servi un prélèvement plus important sur le produit rural, sinon à répandre la misère, sans faire de gagnants. En Nouvelle-France, rien n'incite le capital commercial à accumuler les fermages, à faire main basse sur la rente foncière, à transformer l'hypothèque en propriété. La

36 Voir Marcel Trudel, L'esclavage au Canada français. Histoire et conditions de l'esclavage (Québec, 1960).

37 D'après le mouvement des prix des grains et des marchandises importées, entre 1650 et 1730 . Au prix élevé du blé des débuts a succédé une longue et profonde dépression.

38 L. R. MacDonald, "France and New France: the Internal Contradictions", $C H R$ (juin 1971): 121-143. Le modèle marxiste du passage du capitalisme commercial au capitalisme industriel ne s'applique pas à une petite colonie de l'ancien régime. Il serait plus indiqué de suivre la pensée de Marx sur la formation du capital commercial, appliqué d'abord essentiellement à accroître les déséquilibres régionaux, à élargir les marchés. 
ville n'investit pas dans les campagnes au delà d'une ferme par famille, utile, mais seulement complémentaire. La direction des transferts entre ville et campagnes reste confuse et offre, à maints égards, l'image inversée du schéma traditionnel ${ }^{39}$. Montréal contribue au peuplement rural, car, s'il est vrai que les citadins se transforment difficilement en paysans et optent de préférence pour l'émigration, ces filles de la ville qui épousent des soldats démobilisés ne finissent-elles pas par s'installer dans les côtes ${ }^{40}$ ? La ville ne distribue pas de travail dans les campagnes, hormis ces contrats pour les voyages de traite, incompatibles avec les tâches agricoles. Elle n'apporte guère de capitaux à la terre et les marchands jouent prudemment avec les liens tissés par l'endettement initial des colons. Le dédoublement de l'économie n'est certes pas absolu, les interactions existent, mais elles ne sont pas assez importantes ni pour disloquer, ni pour consolider la société rurale.

Nos conclusions rejoignent celles de tous ceux qui ont réfléchi au problème de la faiblesse économique de la NouvelleFrance et isolé les facteurs fondamentaux: éloignement et insuffisance numérique ${ }^{41}$. Réalités qui persistent après 1760 . La nature du "staple" initial est un élément secondaire. La répartition des profits commerciaux, l'éparpillement social du capital ne sont que des conséquences et n'expliquent pas l'absence d'entreprises de diversification. Les conditions du développement peuvent créer à la longue des attitudes, mais il va sans dire que les facteurs psychologiques ne sont pas des données premières.

Montréal est le lieu de formation d'un capital commercial dont les profits ne sont qu'accessoirement prélevés sur le système de production régional, d'où cette rigidité. Plus tard des facteurs externes vont entraîner ses marchands vers de nouvelles opérations spéculatives, également fondées sur les monopoles et les privilèges, mais qui créent des liaisons plus étroites entre le centre de redistribution et la base territoriale élargie et mieux peuplée. Les disponibilités financières s'accumulent plus vite,

39 Voir les exposés dans G. Friedmann, Villes et Campagnes (Paris, 1953); M. Vénard, Bourgeois et paysans au XVIIe siècle. Recherches sur le rôle des bourgeois parisiens dans la vie agricole au sud de Paris (Paris, 1957), et autres ouvrages.

40 Sur 716 mariages conclus entre 1680 et 1715 dans la paroisse Notre-Dame, $95 \%$ des épouses et $47 \%$ des époux sont Canadiens de naissance.

41 M. H. Watkins, "A Staple Theory of Economic Growth", dans W. T. Easterbrook et Watkins, ed., Approaches to Canadian Economic History, 49-74; P. Harvey, art. cité. 
mais c'est presque un autre siècle qui s'écoule avant que les profits s'effritent suffisamment pour inciter le capital commercial à investir dans la production ${ }^{42}$. Montréal entre alors dans un autre âge.

42 Pour une excellente discussion et illustration de ces problèmes, lire: Pierre Vilar, La Catalogne dans l'Espagne moderne. Recherches sur lob fondements économiques des structures nationales (Paris, 1962). 